\title{
BMJ Open Pulp liner materials in selective caries removal: study protocol for a randomised controlled trial
}

Tássia Carina Stafuzza, ${ }^{1}$ Luciana Lourenço Ribeiro Vitor, ${ }^{1}$ Natalino Lourenço Neto, ${ }^{1}$ Daniela Rios, ${ }^{1}$ Thiago Cruvinel, ${ }^{1}$ Vivien Thiemy Sakai, ${ }^{2}$ Ana Beatriz Silveira Moretti, ${ }^{2}$ Maria Aparecida Andrade Moreira Machado, ${ }^{1}$ Thais Marchini Oliveira (D) ${ }^{1,3}$

To cite: Stafuzza TC, Vitor LLR, Lourenço Neto N, et al. Pulp liner materials in selective caries removal: study protocol for a randomised controlled trial. BMJ Open 2021;11:e029612. doi:10.1136/ bmjopen-2019-029612

- Prepublication history for this paper is available online. To view these files, please visit the journal online (http://dx.doi. org/10.1136/bmjopen-2019029612).

Received 01 February 2019 Revised 21 November 2019 Accepted 03 March 2020
Check for updates

(c) Author(s) (or their employer(s)) 2021. Re-use permitted under CC BY-NC. No commercial re-use. See rights and permissions. Published by BMJ.

${ }^{1}$ Department of Pediatric Dentistry, Orthodontics and Public Health, School of Dentistry of Bauru, University of São Paulo, São Paulo, Brazil ${ }^{2}$ Department of Clinics and Surgery, School of Dentistry, Federal University of Alfenas, Alfenas, Brazil

${ }^{3}$ Department of Pediatric Dentistry, Hospital for the Rehabilitation of Craniofacial Anomalies, University of São Paulo, São Paulo, Brazil

Correspondence to Professor Thais Marchini Oliveira; marchini@usp.br

\section{ABSTRACT}

Introduction The literature shows that selective carious tissue removal (SCTR) decreases the number and diversity of bacteria, stops the caries process and reduces the risk of pulp exposure. However, no consensus exists on which pulp liner would be suitable for teeth undergoing SCTR. So, this study will verify the in vivo response of dentine-pulp complex after SCTR in primary teeth with or without pulp liner material.

Methods and analysis A randomised clinical trial, double-blinded, parallel-group and allocation concealment will be conducted with the enrolment of 384 patients from 5 to 9 years, with one maxillary/mandibular first/second primary molars with deep occlusal/occlusoproximal cavities. The remaining dentine will be lined with calcium hydroxide cement-group 1; mineral trioxide aggregategroup 2 and without liner-group 3. The primary outcome will be success of the of dentine-pulp complex evaluated clinically and radiographically at 6,12 and 24 months, while the secondary outcomes will be the measurement of the dentine barrier on periapical radiographs. During all study, two trained and calibrated examiners will evaluate the treated teeth clinically and radiographically. Interexaminer and intraexaminer reliability will be verified by casual and systematic error. The Kolmogorov-Smirnov test will be adopted to test the normality of continuous variables. Comparisons among groups will be performed by using the $\chi^{2}$ test and anaylsis of variance, followed by Tukey test $(p<0.05)$. The logistic regression will be applied, and the degrees of this association will be measured using the $\mathrm{OR}$ and $95 \% \mathrm{Cl}$.

Ethics and dissemination The present protocol was submitted and approved by the Ethical Committee of the University of São Paulo, Bauru, São Paulo, Brazil (CAAE: 79123517.0.0000.5417). Consent for publication will be obtained from all parents or legal guardians. Results of this study will be reported in full through peer-reviewed journals.

Trial registration number RBR-9fsxnn.

\section{INTRODUCTION}

For deep lesions on teeth with vital pulp, dentists should have as main objective to avoid pulpal exposure by leaving the leathery dentine on the pulp wall. ${ }^{1}$ Within this concept emerged the selective carious tissue removal

\section{Strengths and limitations of this study}

- Report of less invasive management of dental caries focused on promoting and maintaining oral health.

- Provide valuable information to guide the clinical treatment of selective caries tissue removal in primary teeth.

- Randomised clinical trial to compare the clinical and radiographical outcomes after the selective caries tissue removal in primary teeth with or without pulp liner materials.

- To blind the operator during pulp liner application will be not possible.

- The difficulty of selective carious tissue removal is the subjective criterion to evaluate the amount of carious tissue that must be removed in deep lesions.

(SCTR). This treatment changes caries lesion environment because keeping affected dentine decreases the number and diversity of bacteria and stops the caries process. ${ }^{2-4}$ SCTR also allows dentine-pulp complex to deposit tertiary and peritubular dentine, decreasing dentine permeability ${ }^{4}$ and the risk to pulp exposure. ${ }^{5}$

A variety of bacteriostatic, bactericidal, or remineralising materials are applied to the remaining dentine whose effects are discussed in the literature. ${ }^{6}$ The literature has shown that calcium hydroxide cement $(\mathrm{CH})$ can be used as lining material and promotes a decrease in the number of cariogenic bacteria, thus favouring remineralisation. ${ }^{6} 7$ $\mathrm{CH}$ also resulted in clinical and microbiological improvement of the remaining carious dentine in primary teeth. ${ }^{8}$ In contrast, a recent systematic review found a tendency towards failure for teeth lined with $\mathrm{CH}$, despite $\mathrm{CH}$ suitability to treat deep caries, probably because of interactions of factors such as cavity dimension and treatment steps. ${ }^{9}$

Mineral trioxide aggregate (MTA) is a bioactive and insoluble material used as 
protective material for the dental pulp complex of deciduous teeth. ${ }^{10}$ MTA creates an ideal environment for healing, but there are some drawbacks known as a long time of material fixation, high cost and a potential for discolouration. ${ }^{11}$ Some other findings have also indicated that the main goal of sealing the cavity (microbial reduction) after SCTR is achieved regardless of the pulp liner material used. ${ }^{8}$ However, patients with larger amounts of visible plaque are more likely to experience marginal failure in restorations. ${ }^{12}$

To date, the literature lacks consensus on which liner would be suitable for teeth undergoing $\operatorname{SCTR}^{913}$ and claims for long-term randomised controlled studies comparing the clinical and radiographical success rate with other liner materials in paediatric dentistry. ${ }^{14}$ Thus, randomised controlled trials on minimal intervention dentistry and preservation of pulpal vitality would provide clear recommendations to dental practice. ${ }^{10}$

This study will verify whether the use of liner material is necessary after SCTR. This comparison will provide valuable information to guide the clinical treatment of children with deep caries and to train undergraduates, graduates and professionals acting on primary healthcare services. Thus, the aim of this randomised clinical trial will be to verify the in vivo response of dentine-pulp complex after SCTR in primary teeth with or without lining material. The study hypothesis is that the in vivo response of the dentine-pulp complex after SCTR in primary teeth is similar either with or without pulp liner material.

\section{METHODS AND ANALYSIS \\ Study design}

In this double-blinded parallel-group randomised controlled trial, we plan to enrol 384 patients, from 5 to 9 years. Clinical and radiographical success will be assessed after 6,12 and 24 months. Children and their parents will be blinded as to which treatment they will be submitted. Blinding the operator during the liner application step will not be possible. Clinical and radiographical follow-up examinations will be blinded to treatment group allocation. We plan to use the intention-to-treat principle to deal with the data missing at random due imbalanced loss to follow-up by assigning poor outcome to all patients lost in the group that fared better and good outcome to all patients lost in the group that fared worse (extreme case analysis) ${ }^{15}$. During the study, participants who need other dental treatments will be attended by a paediatric dentist at the Pediatric Dentistry Clinic of the University of São Paulo, Bauru, Sao Paulo, Brazil.

\section{Study setting}

Participants will be recruited at municipal schools of Bauru, Sao Paulo Brazil. The children and legal guardians will be instructed about the research and will read and sign a free and clarified consent term. The treatments will take place in the Pediatric Dentistry Clinics of University of Sao Paulo (Bauru, Sao Paulo, Brazil). Efforts will be made to achieve adherence by explaining the treatment importance. Recruitment will take place from September 2019 to August 2020. Each participant will be enrolled in the study for approximately 25 months $(1$ month for treatment plus 24 months of follow-up).

\section{Eligibility criteria}

Inclusion criteria will comprise children aged 5-9 years, from both genders, with restorable maxillary/mandibular first/second primary molars with deep occlusal and occlusoproximal cavities (deep carious lesion reaching pulpal quarter with a zone of dentine separating the lesion from the pulp), ${ }^{16}$ without pulp exposure; no internal/external root resorption, without mobility; no history of spontaneous pain, fistula/abscess and lesion at furcal/periapical area. Exclusion criteria will be the presence of systemic diseases and history of allergic reaction to dental materials. ${ }^{814}$

\section{Allocation and blinding}

Randomisation will be performed after the inclusion of the teeth in the study. The allocation concealment technique will be used to avoid selection bias during the assignment of the selected teeth and the liner material. One molar per patient will be randomly allocated (Microsoft Excel) in the study groups. If the patient has more than one tooth to be included in the study, the tooth mostly meeting the inclusion criteria will be chosen. The selected teeth will be distributed through computerised stratified sampling (Microsoft Excel) according to the child age in months, dmtf index and type of cavity (occlusal or occlusoproximal) ${ }^{17}$ This study will be composed by three groups according to each material: CH (Hydro C/Dentsply, Philadelphia, USA)—group 1 (G1); MTA (Angelus Londrina PR, Brazil)—group 2 (G2) and without liner-group 3 (G3). The following-up period will be 6, 12 and 24 months (figure 1). An equal number of patients will be assigned to the intervention groups.

Randomisation will be independent, that is, the patients and legal guardians will remain blinded to intervention group status. The intervention group identifier will be assigned at random and will not be identified during data analysis, such that data analysts will also remain blinded. This clinical trial follows the guidelines for randomised clinical trials (Standard Protocol Items: Recommendations for Interventional Trials checklist).

\section{Interventions}

Clinical analyses will be based on a form and will be performed by two trained and calibrated operators who will be blinded to the clinical procedure. The operators will be trained on the procedures previously on 10 primary molars that will not be part of the study sample. Radiographical procedures will ensure accurate measurement of reference points, each scanned image will be measured more than once by two trained and calibrated examiners with a 15-day interval between measurements. The 


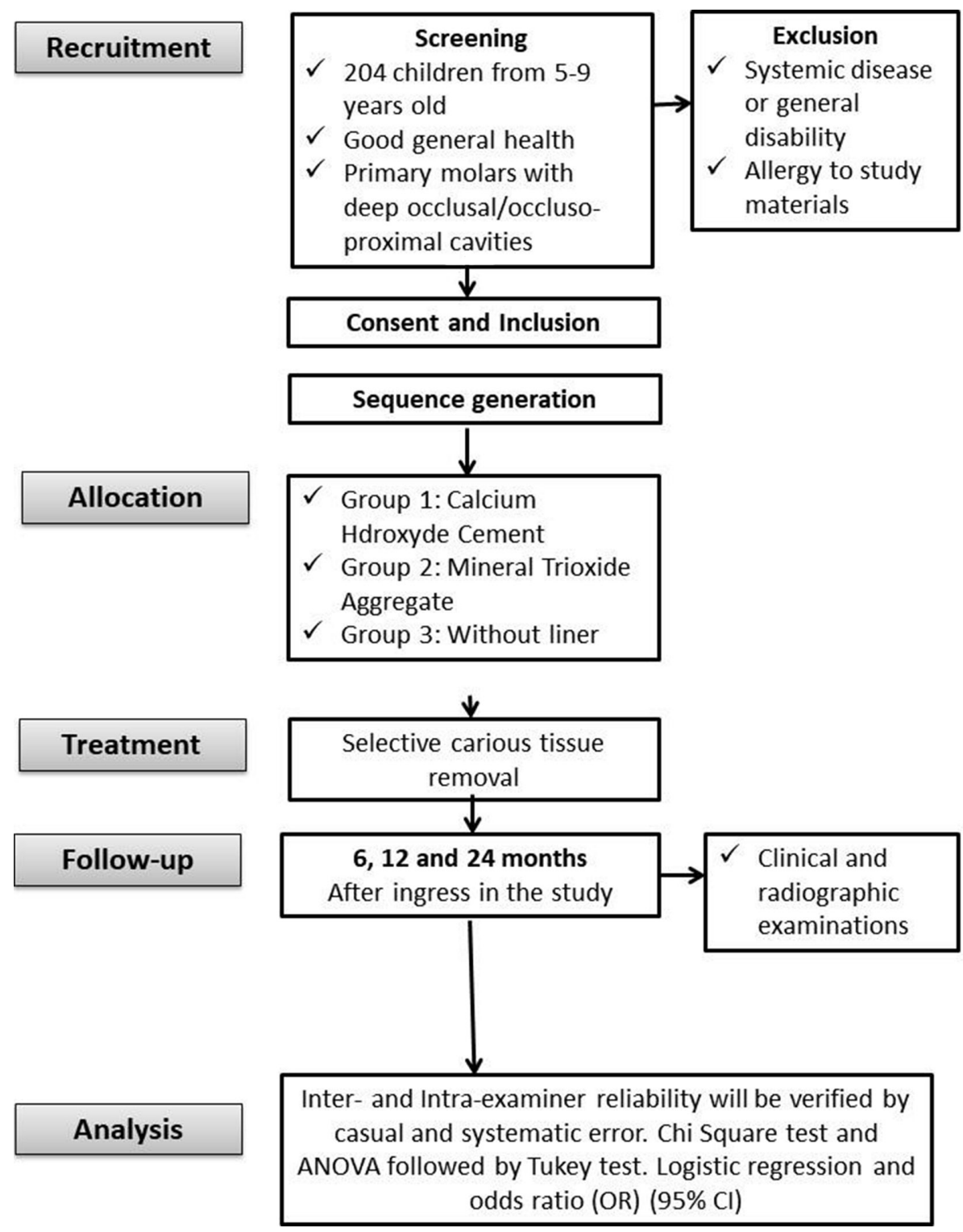

Figure 1 Flowchart of the study. ANOVA, anaylsis of variance

measurements on the digitised images will be performed by two trained and calibrated investigators who will be blinded to the clinical procedure. The patients will be blinded to the pulp liner materials. It will not be possible to blind the operator during pulp liner application. The statistician will be blinded to patient allocation. A detailed anamnesis and clinical examination will be performed. Then a periapical radiograph of the eligible primary molars will be taken to verify the periapical conditions. All radiographs will be standardised using universal acrylic positioning stents. Satisfied all inclusion criteria of the study and after informed consent is given, treatment will be randomly provided in the second visit. After local anaesthesia and rubber dam isolation, the cavity preparation will be performed using water-cooled diamond instruments. Caries will be completely removed from the lateral walls and enamel-dentine junction with the aid of lowspeed round burs until hard dry dentin remains. ${ }^{18}$ SCTR will comprise selective removal to firm dentine on pulpal wall with the aid of hand excavators. The pulp liners will be mixed according to the manufacturers' instructions and gently placed on pulp wall at a thickness of approximately $1 \mathrm{~mm}$. The final restorations will be performed with resin-modified glass ionomer cement (Vitremer 3M/ ESPE). ${ }^{19}{ }^{20}$ If pulpal exposure occurs during excavation, the tooth will be excluded from the study.

\section{Outcomes}

The primary outcome of the study will be success of the in vivo response of dentine-pulp complex (not requiring pulp treatment and maintenance until the normal exfoliation period). The evaluated criteria will include clinical (history of pain, dental mobility, sensitivity to percussion, abscess/fistula and restoration failure) and radiographical aspects (presence/absence of internal/ external resorption, signs of furcal impairment, advanced rhizolysis stage and restoration failure including fracture and loss). Secondary outcome comprises the dentine 
barrier formation. All radiographs will be subsequently digitised and transferred to the computer to be analysed. A baseline radiograph with grid was made with an X-tension C-one P-arallelying (XCP) holder. To assess the dentin barrier thickness, the measurements on the digitised radiographs will be performed at baseline, 6-month, 12-month and 24 month following-up through Corel Draw X3 software (V.13). ${ }^{21}$ To perform the measurement of dentin barrier thickness, the most central point located in the pulp wall of the cavity will be considered. All measurements of the scanned images will be performed by two trained and calibrated investigators who will be blinded to the clinical procedure and the pulp liner materials ${ }^{22}$.

\section{Sample size}

The sample size calculation will be performed based on data from a pilot study. For the sample calculation, alpha and beta errors of $5 \%$ and $20 \%$ will be considered, respectively. The sample size also provides $80 \%$ power. The sample will be of 103 teeth per group to detect significant differences. A drop-out rate of $20 \%$ will be estimated then, the number of eligible teeth will be 128. Thus, the final sample size for this study will be 384 teeth (128 for each study material).

\section{Statistical evaluation}

All data will be analysed using the PASW Statistics V.20 software (SPSS). During all study, two trained and calibrated examiners will evaluate the treated teeth clinically and radiographically. Interexaminer and intraexaminer reliability will be verified by casual and systematic error. The Kolmogorov-Smirnov test will be adopted to test the normality of continuous variables, which will be expressed as median for the variables with a non-normal distribution and a mean value $\pm \mathrm{SD}$ for the variable with a normal distribution. Comparisons among groups will be performed by using the $\chi^{2}$ test and analysis of variance, followed by Tukey test. The level of significance of $5 \%$ will be adopted for all comparisons. The effects of possible confounders (number of involved surfaces, maxillary/ mandibular, first or second molar, age, gender cavity size) will be analysed. The relative cost-effectiveness will be described via the net-benefit approach. The multiple imputation methods will be used to verify sensitivity analysis with regard to missing values. The logistic regression will be applied, and the degrees of this association will be measured via using the OR and 95\% CI.

\section{Trial status}

The trial was registered at ensaiosclinicos.gov.br and the study is open for recruitment in July (). Recruitment will take place from September 2019 to August 2020.

\section{Data collection methods, management and monitoring}

The clinical and radiographical aspects of teeth will be evaluated at 6,12 and 24 months after SCTR procedures. Data will be collected and registered on case report forms by trained researchers blinded to group allocation. Data quality will be ensured by validation checks that include missing data. Clinical and radiographical data will be inserted directly in clinical and radiographical evaluation forms. The data of the scanned X-rays will be entered in Corel Draw X3 software for further analysis. The data will be monitored by an independent clinical research assistant, one statistician and one dental surgeon. No interim analysis beyond that described above is planned. Data monitoring committee (DMC) will independently check data and report any problem. DMC is independent from the sponsor and competing interests.

\section{Ancillary and post-trial care}

After completing the study, participants will continue to receive dental treatments, if needed, in the Pediatric Dentistry Clinics of University of Sao Paulo, Bauru, Sao Paulo, Brazil.

\section{Harms}

It is unlikely that the procedures will result in any adverse effects. The side effects are usually those expected in any conventional dental treatment performed in paediatric dentistry clinical practice.

\section{Patient and public involvement}

Neither patients nor the public will be involved in the design of this study. Our study will only evaluate caries removal treatment in children as described.

\section{Ethics and dissemination}

\section{Ethics approval and consent to participate}

The present protocol was submitted and approved (2018) by the Ethical Committee of the University of São Paulo, Bauru, São Paulo, Brazil (CAAE: 79123517.0.0000.5417). The participants' parents or guardians will receive and sign an informed consent form prior to the child inclusion in the research.

\section{Consent for publication and confidentiality}

All information collected from the participants of the study will be stored in locked filing cabinets in a secure room to protect confidentiality before, during and after the trial. Results of this study will be reported in full through peerreviewed journals. Important protocol modifications will be communicated to the participants, trial register and journals. A model of the informed consent form will be provided, if requested.

\section{Availability of data and materials}

The datasets generated and/or analysed during the current study will be available from the corresponding author on reasonable request.

Acknowledgements The authors would like to thank FAPESP (São Paulo Research Foundation) for the financial support (grant \#2015/13343-9) and the dental clinic staff of the School of Dentistry: Lilian Candida and Estela Ferrari.

Contributors Contributions: TS will carry out the treatments and helped to draft the manuscript; LV will carry out the follow-up evaluation, participated in the study design and helped to draft the manuscript; NLN, DR, TC and ABM will carry out the follow-up evaluation and participated in the study design; VS participated in the study design and helped to draft the manuscript; MAM helped to draft the manuscript; TMO conceived the study, participated in the study design and 
coordination and helped to draft the manuscript. All authors read and approved the final manuscript.

Funding This project had the financial support of the Sao Paulo Research Foundation (FAPESP) grant \#2015/13343-9.

Disclaimer The funder had no role in the study design; collection, management, analysis or interpretation of data; writing of the manuscript or the decision to submit the manuscript for publication.

Competing interests None declared.

Patient consent for publication Not required.

Provenance and peer review Not commissioned; externally peer reviewed.

Open access This is an open access article distributed in accordance with the Creative Commons Attribution Non Commercial (CC BY-NC 4.0) license, which permits others to distribute, remix, adapt, build upon this work non-commercially, and license their derivative works on different terms, provided the original work is properly cited, appropriate credit is given, any changes made indicated, and the use is non-commercial. See: http://creativecommons.org/licenses/by-nc/4.0/.

\section{ORCID iD}

Thais Marchini Oliveira http://orcid.org/0000-0003-3460-3144

\section{REFERENCES}

1 Schwendicke F. Removing carious tissue: why and how? Monogr Oral Sci 2018;27:56-67.

2 Bjørndal L. Indirect pulp therapy and stepwise excavation. J Endod 2008;34:S29-33.

3 Ribeiro CCC, de Oliveira Lula EC, da Costa RCN, et al. Rationale for the partial removal of carious tissue in primary teeth. Pediatr Dent 2012;34:39-41.

4 Ricketts D, Lamont T, Innes NPT, et al. Operative caries management in adults and children. Cochrane Database Syst Rev 2013;3:CD003808.

$5 \mathrm{Li} \mathrm{T}$, Zhai X, Song F, et al. Selective versus non-selective removal for dental caries: a systematic review and meta-analysis. Acta Odontol Scand 2018;76:135-40.

6 Hayashi M, Fujitani M, Yamaki C, et al. Ways of enhancing pulp preservation by stepwise excavation--a systematic review. J Dent 2011;39:95-107.

7 Fernandes JM, Massoni AC, Ferreira JM, et al. Use of calcium hydroxide in deep cavities of primary teeth. Quintessence Int 2013;44:417-23.
8 Bressani AEL, Mariath AAS, Haas AN, et al. Incomplete caries removal and indirect pulp capping in primary molars: a randomized controlled trial. Am J Dent 2013;26:196-200.

9 Schwendicke F, Meyer-Lueckel H, Dörfer C, et al. Failure of incompletely excavated teeth--a systematic review. J Dent 2013;41:569-80.

10 Petrou MA, Alhamoui FA, Welk A, et al. A randomized clinical trial on the use of medical Portland cement, MTA and calcium hydroxide in indirect pulp treatment. Clin Oral Investig 2014;18:1383-9.

11 Parirokh M, Torabinejad M. Mineral trioxide aggregate: a comprehensive literature review--Part III: Clinical applications, drawbacks, and mechanism of action. J Endod 2010;36:400-13.

12 Dalpian DM, Ardenghi TM, Demarco FF, et al. Clinical and radiographic outcomes of partial caries removal restorations performed in primary teeth. Am J Dent 2014;27:68-72.

13 Kidd EAM. Clinical threshold for carious tissue removal. Dent Clin North Am 2010;54:541-9.

14 Schwendicke F, Schweigel H, Petrou MA, et al. Selective or stepwise removal of deep caries in deciduous molars: study protocol for a randomized controlled trial. Trials 2015;16:11.

15 Hollis S, Campbell F. What is meant by intention to treat analysis? survey of published randomised controlled trials. BMJ 1999;319:670-4.

16 Bjørndal L, Simon S, Tomson PL, et al. Management of deep caries and the exposed pulp. Int Endod J 2019;52:949-73.

17 Mendes FM, Pontes LRA, Gimenez T, et al. Impact of the radiographic examination on diagnosis and treatment decision of caries lesions in primary teeth--the Caries Detection in Children (CARDEC-01) trial: study protocol for a randomized controlled trial. Trials 2016;17:69.

18 Innes NPT, Frencken JE, Bjørndal L, et al. Managing carious lesions: consensus recommendations on terminology. Adv Dent Res 2016;28:49-57.

19 Lourenco Neto N, Marques NCT, Fernandes AP, et al. Clinical and radiographic evaluation of Portland cement added to radiopacifying agents in primary molar pulpotomies. Eur Arch Paediatr Dent 2015;16:377-82.

20 Fernandes AP, Lourenço Neto N, Teixeira Marques NC, et al. Clinical and radiographic outcomes of the use of low-level laser therapy in vital pulp of primary teeth. Int J Paediatr Dent 2015;25:144-50.

21 George V, Janardhanan SK, Varma B, et al. Clinical and radiographic evaluation of indirect pulp treatment with MTA and calcium hydroxide in primary teeth (in-vivo study). J Indian Soc Pedod Prev Dent 2015;33:104-10.

22 Leye Benoist F, Gaye Ndiaye F, Kane AW, et al. Evaluation of mineral trioxide aggregate (MTA) versus calcium hydroxide cement (Dycal(囚)) in the formation of a dentine bridge: a randomised controlled trial. Int Dent J 2012;62:33-9. 